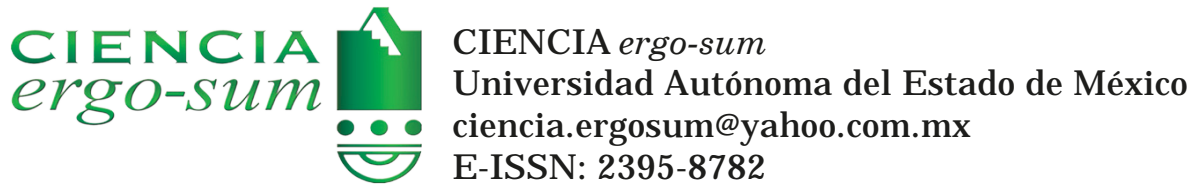

\title{
Resiliencia en adolescentes y adultos en internamiento por diversos delitos
}

Vargas Sánchez, Karla María; González Arratia López Fuentes, Norma I vonne; Valdez M edina, J osé Luist;
González Escobar, Sergio; Zanatta Colín, Elizabeth
Resiliencia en adolescentes y adultos en internamiento por diversos delitos
CIENCIA ergo-sum, vol. 27, núm. 1, marzo-junio 2020| e72
Universidad Autónoma del Estado de México, México
Esta obra está bajo una Licencia Creative Commons Atribución-NoComercial-SinDerivar 4.0 Internacional.

Vargas Sánchez, K. M., González Arratia López Fuentes, N. I., Valdez M edina, J . L., González E scobar, S., Zanatta Colín, E. (2020). Resiliencia en adolescentes y adultos en internamiento por diversos delitos. CIE NCIA ergo-sum, 27(1). https://doi.org/10.30878/ces.v27n1a4 


\title{
Resiliencia en adolescentes y adultos en internamiento por diversos delitos
}

The resilience of teenagers and adults in internment for various crimes

\author{
Karla Maria Vargas Sánchez \\ Universidad Autónoma del Estado de México, México \\ lic.vargas_04@hotmail.com \\ Norma Ivonne González Arratia López Fuentes \\ Universidad Autónoma del Estado de México, México \\ nigalf@yahoo.com.mx \\ José Luis Valdez Medina ${ }^{\dagger}$ \\ Universidad Autónoma del Estado de México, México \\ Sergio González Escobar \\ Universidad Autónoma del Estado de México, México \\ sergioglz4@hotmail.com \\ Elizabeth Zanatta Colin \\ Universidad Autónoma del Estado de México, México \\ elizatt12@hotmail.com
}

Recepción: 16 de abril de 2018

\section{RESUMEN}

Se exploran las características de resiliencia en personas privadas de la libertad, así como los factores protectores internos, externos y de riesgo. Participaron 292 personas internas en instituciones penitenciarias del Estado de México, quienes fueron evaluadas con una escala de resiliencia que consta de dos partes. De la primera, se obtuvo mayor frecuencia en el nivel moderado de resiliencia (60\% de adolescentes y $62 \%$ de adultos); en la complementaria, se reporta el internamiento y la pérdida de un familiar como los sucesos más difíciles de superar, además del apoyo de la familia como el factor protector externo más importante en su condición actual. Contar con características de resiliencia permite amortiguar el impacto del internamiento y adaptarse al funcionamiento institucional.

Palabras claVe: resiliencia, instituciones penitenciarias, hombres, mujeres.

\begin{abstract}
The resilience characteristics of people in deprivation of liberty as well as the internal, external and risk protective factors were studied. A sample of 292 people from different penitentiaries in the State of Mexico was evaluated with a two-part resilience scale. The first part reflects a moderated level of resilience (60\% teenagers and $62 \%$ adults); in the complementary part, internment and the loss of a family member are reported to be the most difficult situations to overcome; besides, inmates recognize the family support as the most important external factor in their internment condition. It is important to have characteristics of resilience in a penitentiary context to lessen the internment impact and to be able to adapt to the institutional functioning.

KEYWORDS: resilience, penitentiaries, men, women.
\end{abstract}

\section{INTRODUCCIÓN}

El concepto de resiliencia surge en la metalurgia y se refiere a la capacidad de los metales de resistir un impacto y recuperar su estructura. En psicología, el concepto se basa en acciones que favorecen el crecimiento de una 
persona a partir de eventos o situaciones de adversidad. Correspondería a la capacidad del ser humano de hacer frente a las adversidades de la vida, superarlas y salir fortalecido de ellas o incluso transformado (Grotberg, 2006; Saavedra, 2009; González-Arratia, 2011).

El vocablo resiliencia tiene su origen en el latín resilio que significa "volver atrás, volver de un salto, rebotar". Este término fue adoptado por las ciencias sociales para caracterizar a aquellas personas que a pesar de nacer y vivir situaciones de alto riesgo se desarrollan psicológicamente sanas y exitosas (Córdova, 2006).

De manera tradicional se ha estudiado la enfermedad y el trauma, así como las consecuencias en la salud mental de los individuos. Sin embargo, desde la psicología positiva, en la cual se enmarca este artículo, se busca entender los procesos y mecanismos para el crecimiento personal y fortalecimiento de habilidades (Montelongo, 2013). Por ello, se concibe al individuo no sólo como una víctima de las circunstancias, sino como un ser activo con capacidad de resistir y rehacerse a pesar de la adversidad (Vera, et al., 2006).

La resiliencia ha sido definida de muchas maneras, entre ellas, como un proceso o capacidad de adaptación. Luthar (1993) la conceptualiza como la capacidad del ser humano de recuperarse de la adversidad y de trasformarla en un elemento de estímulo y desarrollo. Posteriormente, se consideró que la resiliencia no implicaba sólo una capacidad humana, sino que iba acompañada de otros factores que favorecerían su desarrollo en el individuo, por lo que se entiende como un proceso dinámico entre el individuo y su contexto, el cual tiene como resultado la adaptación y manejo adecuado en situaciones de gran adversidad (Luthar et al., 2000, en González Arratia, 2011).

Si bien no existe un consenso en su definición, desde el enfoque ecológico la resiliencia es dinámica. Para fines de esta investigación, se entiende que es la capacidad de los individuos para adaptarse o ajustarse de manera constructiva a pesar de encontrarse en una situación adversa o de crisis (González Arratia, 2018). Implica la combinación o interacción entre los atributos del individuo (internos) y su ambiente familiar, social y cultural (externos) que lo posibilitan a superar el riesgo y la adversidad de forma constructiva (González Arratia, 2007).

Esta definición se sustenta desde el modelo de Bronfenbrenner $(1979,2000)$ en el cual se propone una visión relacional y contextual del desarrollo humano enfatizando en la interacción dinámica persona-ambiente desde cinco niveles o sistemas anidados (macrosistemas, mesosistemas, exosistemas, macrosistema, cronosistema) que pueden verse reflejados como aquellos factores externos con los cuales cuenta o contará el individuo para mitigar aquellas situaciones de adversidad en donde se vea involucrado.

De ahí la importancia de que la resiliencia deba concebirse como un constructo multidimensional reforzado por autores como Luthar (1993), Masten, et al. (1990), Kaplan (1999) y Bernard (1999), donde se reconoce que la adopción del enfoque psicoecológico tiene precedentes de aplicación a los estudios de resiliencia identificándola como un proceso dinámico donde las influencias ambientales y del individuo interactúan en una relación recíproca entre ambos (González Arratia, 2007).

Según Mikulic y Crespi (2004: 212) "a la situación de privación de libertad, se le puede considerar como una transición ecológica que trastoca el modo habitual de ser y de estar de los sujetos y que conduce a la adopción de nuevas pautas de conductas, de roles y de modalidades relacionales, como forma de adaptarse a los nuevos requerimientos y demandas del contexto carcelario".

Al presentar un enfoque multidimensional y dinámico, como ya se ha mencionado, es importante considerar el contexto en donde se desenvuelve un individuo, así como el impacto que éste le conlleve; dicho lo anterior, resulta pertinente preguntarse: ¿un individuo puede presentar características resilientes en un contexto penitenciario?

La situación de reclusión impacta de manera directa en la integridad y adecuación de los individuos privados de su libertad, así como de aquellas personas que las rodean (CNDH, 2013). Al respecto, se ha reportado que las condiciones de estancia de las personas que se encuentran en centros preventivos, requieren de determinadas estrategias de afrontamiento que van a depender de la evaluación que ellos mismos realicen de tales situaciones (Lazarus y Folkman, 1991) y principalmente de las características de los estresores o eventos adversos a los que se enfrenten, lo cual puede hacer que la estancia en estos contextos se convierta 
en un conjunto de factores que pueden considerarse de adversidad para el individuo desencadenando en él un deterioro tanto físico como psicológico.

De lo anterior, se deriva la importancia de identificar si las personas que se encuentran en situación de encarcelamiento de algún modo manifiestan conductas que favorezcan la adaptación al nuevo medio donde se desenvuelven. Esto resulta relevante debido a que se ha demostrado que la privación de la libertad incrementa el estrés percibido por los detenidos en los diversos dominios de su vida como lo es la familia y la pareja, además de que disminuyen los recursos psicológicos con los que cuenta el sujeto (Mikulic y Crespi, 2004).

Siguiendo esta línea, Hernández (2008) y Ramírez (2008) han realizado investigaciones sobre resiliencia en adolescentes que se encuentran en internamiento y en donde han identificado que existen características resilientes en los menores infractores, además de alta autoestima. A su vez, Ramírez (2008) identificó diferencias estadísticamente significativas relacionadas con el género, donde las mujeres tienden a ser más resilientes que los hombres.

En otro estudio realizado en México con adolescentes en internamiento, Hernández (2009) refiere que, a pesar de presentar graves problemas de conducta, cuando se encuentran en condiciones favorables dentro de su entorno, son capaces de dar mejores resultados en cuanto a una rehabilitación puesto que desarrollan cualidades como la de ser sensibles, tranquilos, trabajadores, agradecidos y generosos.

Lo anterior evidencia que, independientemente de las características que haya presentado el adolescente al momento de ingresar al tutelar, un ambiente favorable acompañado del tratamiento institucional podría favorecer la manifestación de características positivas en su comportamiento que ser parte de la estructura de su personalidad y que les permita una reintegración favorable en la sociedad.

Los estudios son escasos respecto a la población adulta que se encuentra recluida y a la resiliencia, pero se ha encontrado evidencia en países como Chile, Colombia y Argentina; en este último, por ejemplo, existen antecedentes de investigación, donde se ha identificado que las características resilientes entre personas presas y personas que no lo hayan estado eran similares y que al mismo tiempo podrían considerarse como escasas (Grieco, 2012), lo cual podría ser a causa de las condiciones de riesgo del medioambiente social y familiar donde se desenvuelven y que no favorecen la posibilidad de ser resilientes.

De igual forma, Vargas (2010) reporta que la mayoría de las personas privadas de la libertad por cometer algún delito presentaron un nivel alto de resiliencia y, en menor medida, encontró individuos con características de resiliencia moderada y baja. Esto evidencia que a pesar de vivir en reclusión los individuos tienden a presentar características resilientes que les pueden ayudar a mitigar el impacto de vivir en una prisión.

En cuanto al sistema penitenciario, la situación en México es compleja, ya que de los 420 centros penitenciarios 220 presentan sobrepoblación, lo cual refleja un 47.47\% de esta problemática; específicamente en el Estado de México, 22 centros reportan 70.48\% de sobrepoblación (INEGI, 2013). Lo anterior conlleva condiciones precarias para las personas que se encuentran recluidas, como el hacinamiento y la sobreproblación. Por ello, surge la relevancia de retomar estudios con el fin de analizar las características de resiliencia dentro de contextos penitenciarios que puedan ser útiles para implementar estrategias de intervención y así permitir la reintegración social.

La adaptación a la vida en prisión como interno o interna se expresa en aspectos cognitivos, emocionales, comportamentales y socioculturales. Tal adaptación va a estar mediada por experiencias previas al encierro (Becerra et al., 2008). Estas experiencias tienen que ver con la historia de vida de las personas, específicamente de aquellas que el individuo percibe como de alto impacto o adversidad y cómo las resolvieron o afrontaron.

Se debe considerar también que el internamiento para una persona implica un aislamiento afectivo y social, además de la pérdida de sus roles sexuales, familiares y sociales causando en ella un deterioro en su identidad y autoestima. Es importante identificar aquellas conductas que se manifiestan cuando la persona se encuentra ante esta situación, puesto que se ve sometida a un ritmo de horario y circunstancias no elegidas por él o ella que en muchos casos influye negativamente hasta en su salud (física y psíquica) y le exige adaptarse a un nuevo medio que en la mayoría de los casos es percibido como desfavorable y hostil (Rubio, 2013). 
A partir de investigaciones se ha identificado que la reclusión implica para un ser humano la disminución general de su repertorio de conducta por efecto de la estancia prolongada en el centro penitenciario (Clemmer, 1940; Goffman, 1979; Pérez y Redondo, 1991; Pinatel, 1969, en Herrera y Expósito, 2010).

Ahora bien, considerando la variable sexo, Lagarde (1993, en Herrera y Expósito, 2010) acotan que las mujeres sufren más la prisión que los hombres, ya que su encarcelamiento afecta directamente su núcleo familiar, pues ellas suelen ser el soporte de la familia tanto afectivo como económico, es decir, sufren una doble condena: la del encierro y la social.

El ingreso en prisión de una mujer, además de lo ya referido, conlleva el estigma familiar, alteración de los roles y problemas de apego que se ven agravados en el caso de tener hijos e hijas pequeños en prisión (Lagarde, 1993 en Herrera y Expósito, 2010).

Las tasas de ira, depresión, ansiedad y grado de control no cambian con el paso del tiempo, como tampoco lo hace la tolerancia a los estresores y el uso de tipos de afrontamiento (Paulus y Dzindolet, 1993 en Becerra et al., 2008). Es decir, de contar con características positivas, éstas no se verán mitigadas ante un estado de reclusión, sino que permanecerán dando pie a la posibilidad de que si el individuo cuenta con ellas, las manifieste estando en prisión.

En la misma tesitura el apoyo social es un factor que actúa como protector frente a la sintomatología de estrés y ansiedad (propias de un estado de reclusión) y se asocia directamente con estilos de afrontamiento como el activo y la búsqueda de apoyo (Ruiz, 2004, en Becerra et al., 2008), estilos considerados como positivos y que se correlacionan con las características resilientes en una persona.

Por lo expuesto, es importante identificar si los individuos que se encuentran internos en instituciones penitenciarias presentan características resilientes que les permitan adaptarse y sobrellevar la reclusión, así como identificar si el sexo y la edad determinan en mayor o menor medida el nivel de resiliencia.

Cabe mencionar que para fines de esta investigación la población estuvo conformada por adolescentes y adultos, lo cual es importante de precisar, ya que los estudios acerca de la resiliencia han sido enfocados principalmente a niños y adolescentes y han excluido a la población adulta en estos contextos, y que sin embargo es importante estudiar porque la condición de ser adulto no lo exenta de vivir situaciones de adversidad o alto estrés.

El interés en el tema de la resiliencia significa un reto y al mismo tiempo un desafío para que los estudiosos de psicología dispongan de conocimiento sobre investigación en un sentido positivo (González-Arratia y Valdez, 2012), lo cual sea por medio de la identificación y la propuesta de alternativas distintas a las convencionales en lo que corresponde a la intervención e investigación psicológica no sólo para ayudar a resolver los problemas de salud mental que adolecen los individuos, sino también para alcanzar mejor calidad de vida y bienestar individual, independientemente del contexto en el que se desenvuelvan.

Por lo anterior, se parte de la consideración de que la vida en reclusión exige el uso de los recursos psicológicos del individuo, entre los cuales se puede considerar a la resiliencia, que podría ya poseer y que permitirán la adaptación a la dinámica en este tipo de contextos.

\section{MÉTodo}

Se llevó a cabo un estudio descriptivo que busca especificar las propiedades, las características y los perfiles importantes de personas, grupos, comunidades o cualquier otro fenómeno que se someta a análisis (Danhke, 1989 en Hernández et al., 2003). Específicamente, este estudio tiene un corte mixto, puesto que se consideraron aspectos cualitativos y cuantitativos para una mayor comprensión de la resiliencia.

\section{1. Participantes}

Una vez obtenida la autorización por parte de las instituciones penitenciarias, y previo consentimiento informado de los participantes, se trabajó con una muestra total de 292 personas, de las cuales 214 son adultos ( 107 hombres 
y 107 mujeres, edad $\overline{\mathrm{x}}=32.31$ ) y 78 adolescentes (50 hombres y 28 mujeres, edad $\overline{\mathrm{x}}=17.50$ ), quienes al momento de la evaluación se encontraban internas por diversos delitos como homicidio, secuestro, robo con violencia, delincuencia organizada, robo de infante, daños contra la salud y violación en diferentes instituciones de readaptación social pertenecientes al Estado de México. En virtud de que era una gama amplia de delitos y debido a la preservación de confidencialidad, no fue posible clasificar la muestra por delitos. Asimismo, por cuestiones institucionales no se permitió obtener datos sociodemográficos como estado civil y lugar de origen.

\subsection{Instrumento}

Escala de resiliencia (González Arratia, 2011), la cual explora la capacidad humana que posibilita al individuo a interactuar en el medio y ajustarse a los cambios y demandas de diferentes eventos de manera positiva. Consta de 32 reactivos compuestos por tres factores: a) Factores protectores internos: son aquellos atributos del individuo (reactivos 17,18, 20, 21, 23, 24, 25, 26, 27, 28, 29, 30, 31 y 32). b) Factores protectores externos: abarcan el ambiente familiar, social y cultural (reactivos 1, 4, 5, 6,10, 11, 12, 13, 14, 15 y 16. c) Empatía: se refiere al comportamiento altruista y prosocial (reactivos 2, 3, 7, 8, 9, 19 y 22). Con cinco opciones de respuesta de tipo Likert: siempre, la mayoría de las veces, indeciso, algunas veces y nunca, donde el puntaje mínimo es de 32 y el máximo de 160, además consta de un $40.30 \%$ de varianza y una Alfa de Cronbach de 0.9352.

La parte complementaria está compuesta por un cuestionario abierto de resiliencia conformado por 10 preguntas abiertas (González Arratia et al., 2008); en él se indaga acerca de eventos que el individuo considera difíciles o adversos, cómo los afronta, cómo se siente respecto a ellos, a quién acude ante dichos eventos y las expectativas a futuro. Este cuestionario complementa la escala de resiliencia (González Arratia, 2011) al aportar datos de corte cualitativo.

\subsection{Procesamiento de información}

Para el análisis de la escala, se utilizó el programa SPSS versión 17 y se realizaron análisis descriptivos. Posteriormente, se aplicó la prueba $t$ de Student para muestras independientes y así observar diferencias en cuanto a las medias de los grupos a partir de la variable sexo y edad con un nivel de significancia de $p=0.05$ y un nivel de confianza de $95 \%$.

Para la escala complementaria, se hizo un análisis de contenido a partir de las respuestas de los individuos y mediante un jueceo de expertos se determinaron seis categorías (familiar, personal, conductual, social, física/ salud y afectiva). De lo anterior, se obtuvieron análisis de frecuencias de cada pregunta obteniendo de este modo los datos más representativos de la muestra.

\section{Resultados}

Se presentan datos de la escala de resiliencia (González Arratia, 2011) donde para obtener el nivel de ésta, del puntaje total, se establecieron puntos de corte a partir de la media de +/- 1 desviación estándar; con base en ello se obtuvieron tres niveles: bajo (puntajes menores a 120), moderado (puntajes entre 121-149) y alto (puntajes mayores a 150).

De acuerdo con este criterio, se reporta que $23 \%$ de la muestra de adolescentes y $21 \%$ de adultos puntuaron en el nivel alto de resiliencia, $60 \%$ de los adolescentes y $62 \%$ de adultos en el nivel moderado y $17 \%$ de los adolescentes y $21 \%$ de los adultos en el nivel bajo. Las medias indican que los hombres, tanto adolescentes como adultos mostraron la media más alta en el nivel de resiliencia (cuadro 1). 


\section{CUADRO 1}

Datos descriptivos (nivel de resiliencia en adolescentes y adultos recluidos)

\begin{tabular}{|lcccrrr}
\hline & \multicolumn{3}{c}{$\begin{array}{c}\text { Nivel de resiliencia } \\
\text { Adolescentes }\end{array}$} & \multicolumn{3}{c|}{$\begin{array}{c}\text { Nivel de resiliencia } \\
\text { Adultos }\end{array}$} \\
\hline & Alto & Moderado & Bajo & Alto & Moderado & \multicolumn{1}{c}{ Bajo } \\
\hline Media Hombres & 150 & 132.08 & $\mathbf{1 1 1 . 5 0}$ & 156.33 & 140.36 & $\mathbf{1 1 5 . 8 2}$ \\
DE & 2.66 & 7.53 & 4.50 & 2.49 & 7.69 & 7.82 \\
Media Mujeres & $\mathbf{1 5 1 . 7 1}$ & $\mathbf{1 3 3 . 7 4}$ & 108.50 & 155.92 & $\mathbf{1 4 0 . 4 4}$ & 113.38 \\
DE & 3.25 & 8.52 & 4.95 & 2.18 & 8.59 & 8.68 \\
\hline \multicolumn{5}{c}{ Fuente: elaboración propia } \\
\hline \multicolumn{4}{c}{}
\end{tabular}

Asimismo, en cuanto a la dimensión de resiliencia medida con la escala de González Arratia (2011) se analizaron las diferencias con respecto al sexo: para ello se realizó una prueba $t$ de Student, donde se obtuvo una diferencia estadísticamente significativa a favor de las mujeres en la muestra de adolescentes, es decir, las mujeres en este grupo de edad tienden a ser más resilientes que los hombres. En cuanto a la muestra de adultos, no se obtuvo diferencia estadísticamente significativa (cuadro 2).

\section{CUADRO 2}

Resiliencia en adolescentes y adultos recluidos (diferencias según sexo)

\begin{tabular}{|c|c|c|c|c|c|c|c|c|c|c|}
\hline & \multicolumn{5}{|c|}{ Muestra adolescente } & \multicolumn{5}{|c|}{ Muestra adultos } \\
\hline & $\begin{array}{l}\text { Media } \\
\text { Hombres } \\
\mathrm{N}=50\end{array}$ & $D E$ & $\begin{array}{l}\text { Media } \\
\text { Mujeres } \\
\mathrm{N}=28\end{array}$ & $D E$ & $t$ & $\begin{array}{l}\text { Media } \\
\text { Hombres } \\
\mathrm{N}=107\end{array}$ & $D E$ & $\begin{array}{l}\text { Media } \\
\text { Mujeres } \\
\mathrm{N}=107\end{array}$ & $D E$ & $t$ \\
\hline $\begin{array}{l}\text { Puntaje total } \\
\text { de resiliencia }\end{array}$ & 129.9 & 14.7 & 136.4 & 13.2 & $1.94^{*}$ & 137.5 & 14.3 & 138.4 & 15.8 & 0.4 \\
\hline
\end{tabular}

Fuente: elaboración propia.

Nota: ${ }^{*} p<0.05$.

En lo correspondiente a la información obtenida a través del cuestionario abierto de resiliencia, para la muestra adolescente y adultos, se analizó su contenido del cual se obtuvieron cinco categorías: área personal, conductual, social, física/salud y afectiva; posteriormente, se realizó un análisis de frecuencias y se agrupó dentro de los factores que se relacionan con la resiliencia (internos, externos y de riesgo). En el cuadro 3 se puede observar que entre los factores de riesgo se reportaron con mayor frecuencia factores sociales (estar en internamiento), factores familiares (pérdida de un ser querido, separación de la familia) y factores conductuales (comisión de delito), de los cuales las mujeres refieren con mayor frecuencia factores de riesgo sociales y familiares y en cuanto a los hombres se reportan factores sociales y conductuales.

Respecto a los factores protectores internos, se mencionaron situaciones que tienen que ver con la visión positiva de sí mismo, las cuales incluyen la seguridad en sí mismo o misma y estrategias de afrontamiento y autonomía, donde los hombres refieren más características que tienen que ver con las estrategias de afrontamiento y las mujeres con el valor de sí misma y la autonomía (cuadro 4).

En lo referente a los factores protectores externos se reportan factores familiares (padres, pareja, hermanos, tíos/tías), sociales (amigos, compañeros, personas de confianza) y otras redes de apoyo (centros de autoayuda, psicólogo, profesores). En cuanto al factor familiar, las mujeres indican que acuden a sus familiares y los hombres además a la pareja; en el ámbito social, las mujeres mencionan a los amigos como red de apoyo y los hombres a las personas de confianza; finalmente, entre los otros tipos de redes de apoyo las mujeres acuden en mayor medida a los profesionales y a los centros de autoayuda que los hombres (cuadro 5). 


\section{CUADRO 3}

Frecuencias de los factores de riesgo (población adulta y adolescente)

\begin{tabular}{|c|c|c|c|c|c|c|}
\hline \multirow{2}{*}{ Categoría } & \multirow{2}{*}{ Indicador } & \multicolumn{2}{|c|}{ Adultos } & \multicolumn{2}{|c|}{ Adolescentes } & \multirow{2}{*}{ Frecuencia Total } \\
\hline & & $\mathbf{H}$ & $\mathbf{M}$ & $\mathbf{H}$ & M & \\
\hline Personal & Encontrarse recluido & 35 & 55 & 15 & 21 & 126 \\
\hline \multirow{2}{*}{ Familiar } & Pérdida de un familiar & 8 & 9 & 3 & - & \multirow{2}{*}{29} \\
\hline & Estar lejos de la familia & 2 & 6 & - & 1 & \\
\hline Social & Dificultad para relacionarse & 6 & 5 & 1 & 1 & 13 \\
\hline Conductual & $\begin{array}{l}\text { Cometer un delito (robo, } \\
\text { violación, homicidio y } \\
\text { secuestro) }\end{array}$ & 3 & - & 6 & 1 & 10 \\
\hline Física & $\begin{array}{l}\text { Enfermedad/consumo de } \\
\text { sustancias }\end{array}$ & 3 & - & 5 & - & 8 \\
\hline Laboral & Trabajar a temprana edad & 2 & - & 1 & - & 3 \\
\hline
\end{tabular}

Fuente: elaboración propia.

Nota: se reportan únicamente los indicadores de mayor frecuencia.

\section{CUADRO 4}

Frecuencias de los factores protectores internos (población adulta y adolescente)

\begin{tabular}{|c|c|c|c|c|c|c|}
\hline \multirow{2}{*}{ Factor Protector Interno } & \multirow{2}{*}{ Indicador } & \multicolumn{2}{|c|}{ Adultos } & \multicolumn{2}{|c|}{ Adolescentes } & \multirow{2}{*}{$\begin{array}{c}\text { Frecuencia } \\
\text { Total }\end{array}$} \\
\hline & & $\mathbf{H}$ & $\mathbf{M}$ & $\mathbf{H}$ & $\mathbf{M}$ & \\
\hline \multirow[t]{3}{*}{ Visión positiva de sí mismo } & Seguridad en sí mismo & 39 & 40 & 15 & 5 & 99 \\
\hline & Estrategias de afrontamiento & 33 & 19 & 16 & 6 & 74 \\
\hline & Autonomía & 2 & 12 & 4 & 1 & 19 \\
\hline
\end{tabular}

Fuente: elaboración propia.

\section{CUADRO 5}

Frecuencias de los factores protectores externos (población adulta y adolescente)

\begin{tabular}{|c|c|c|c|c|c|c|}
\hline \multirow{2}{*}{ Categoría } & \multirow{2}{*}{ Indicador } & \multicolumn{2}{|c|}{ Adultos } & \multicolumn{2}{|c|}{ Adolescentes } & \multirow{2}{*}{$\begin{array}{c}\text { Frecuencia } \\
\text { Total }\end{array}$} \\
\hline & & $\mathbf{H}$ & $\mathbf{M}$ & $\mathbf{H}$ & M & \\
\hline Familiar & Padres, hermanos, tíos/tías y pareja & 28 & 24 & 24 & 8 & 84 \\
\hline Social & Amigos y personas de confianza & 18 & 17 & 8 & 11 & 54 \\
\hline Otro & Centros de autoayuda y profesionales & 14 & 21 & - & - & 35 \\
\hline
\end{tabular}

Fuente: elaboración propia. 


\section{Discusión}

A partir de los resultados cuantitativos obtenidos, se encontró en mayor medida que los individuos se ubican en un nivel de resiliencia moderado, lo cual evidencia la presencia de recursos psicológicos positivos que posiblemente permitirán que el individuo logre afrontar el estado de reclusión de manera funcional; esto se sustenta con estudios respecto a la resiliencia, donde se enfatiza que ser resiliente es un indicador favorable para los individuos, pues les permite desarrollarse de manera adecuada en distintos ámbitos (González-Arratia y Valdez, 2012).

De acuerdo con las diferencias por sexo, se reporta que las mujeres adolescentes tienden a ser más resilientes en comparación con los hombres adolescentes; esta tendencia ya se ha reportado en estudios anteriores, donde se identifican características resilientes con mayor frecuencia en las mujeres que también se encuentran en internamiento (Ramírez, 2008).

En cuanto a la población adulta, no se encontró diferencia estadísticamente significativa respecto al sexo, condición referida por Saavedra y Villalta (2008). Estos autores coinciden en que no existe diferencia respecto a ser hombre y mujer en los niveles de resiliencia, pero que ésta tiende a manifestarse de formas distintas en un hombre y una mujer, esto es, que de existir las características resilientes se manifestarán de forma distinta según cada individuo; el resultado coincide con el obtenido en el presente estudio a través del cuestionario abierto, donde los hombres refieren características que tienen que ver con las estrategias de afrontamiento y las mujeres con el valor de sí misma , la autonomía y el apoyo social, especialmente de la familia.

Los factores de riesgo se refieren a situaciones contextuales o personales que al estar presentes incrementan la probabilidad de desarrollar problemas emocionales, conductuales o de salud (Hein, 2004, en González-Arratia, 2011). Así, dentro de los resultados cualitativos, los individuos reportaron en la escala complementaria de resiliencia que en mayor medida la situación de encontrarse recluido es un factor de riesgo ante la posibilidad de experimentar problemas emocionales, lo cual se corrobora con lo documentado acerca de la situación que viven las personas que se encuentran privadas de la libertad en función de que las instituciones penitenciarias tienen una serie de peculiaridades dentro de su dinámica que favorece que la estancia en ellas se convierta en una situación de suma adversidad.

En la misma línea se manifiestan como factores de riesgo las situaciones familiares que implican la pérdida de un ser querido y el estar alejado de la familia, también se reportaron factores conductuales como lo es la comisión de un delito, las cuales son percibidas por las personas como situaciones difíciles de experimentar y afrontar.

En lo que se refiere a la población adolescente las situaciones de adversidad corresponden, por ejemplo, a presenciar peleas de sus padres, involucrarse en organizaciones delictivas, el consumo de drogas y el hecho de haber lastimado a terceras personas. Un aspecto importante a destacar es que en las mujeres el encontrarse alejadas de los hijos, no poder cuidarlos o un embarazo, son situaciones que perciben como adversas en el momento de estar en internamiento, mismas que se correlacionan con factores socioculturales.

Enfrentar o experimentar las situaciones mencionadas conlleva un impacto en el individuo a nivel personal, toda vez que manifiestan tener conflicto consigo mismos, problemas en su autoestima y se perciben incapaces de resolver problemas, así como a nivel familiar, puesto que surgen conflictos familiares, temor de ser abandonados por su familia, falta de comunicación entre sus integrantes y sentir impotencia al no estar en posibilidad de ayudar en la crianza de los hijos. Con base en esto, es posible aseverar que la situación de reclusión debe ser considerada como un evento de alto impacto, estrés y adversidad para el ser humano, la cual exige recursos psicológicos para sobrellevarla o adaptarse.

Por otro lado, respecto a los factores protectores que los individuos manifiestan después de la presencia de algún estresor o experiencia adversa, se identifica una modificación de la respuesta del individuo, en un sentido comparativamente más adaptativo que el esperado o más utilizado (Rutter, 1985, en González-Arratia, 2011). Lo anterior explicaría el motivo por el cual los individuos refieren que posterior a las vivencias de adversidad 
(la reclusión, separación familiar) se percibieron más fuertes, se dieron cuenta que pueden solucionar problemas valiéndose de sus estrategias de afrontamiento, que son capaces de "salir adelante" siendo perseverantes, a ser tolerantes, a ser responsables de sus actos y valorarse a sí mismos, además de que los adolescentes refieren percibirse más independientes. Todo lo anterior es lo que corresponde a los factores protectores internos, es decir, aquellos que son propios del individuo y conllevan elementos intrínsecos.

$\mathrm{Y}$ en lo que se refiere a los factores protectores externos, los individuos reportan que el apoyo de la familia, amigos, de psicólogos/maestros dentro de las instituciones y de la pareja, auxilia y favorece a la superación de las situaciones de adversidad que se le presentan; en el caso de las mujeres resalta el apoyo por parte de los hijos como un factor importante para superar situaciones difíciles.

Por lo expuesto, es posible corroborar la importancia que tienen las redes de apoyo para que el individuo se sienta capaz de resolver experiencias adversas o difíciles. Si se consideran estos elementos para la intervención institucional, se podría fomentar y motivar aquellos factores existentes en el individuo que darán paso a una funcionalidad y adaptación más adecuada a las dinámicas institucionales, e incluso a una posible y real reinserción social.

\section{Conclusiones}

Con base en los resultados obtenidos, se encontró que los individuos resilientes presentan habilidades individuales y redes de apoyo (como familia, amigos y psicólogos) que facilitan o mitigan el impacto que se tiene ante la situación de internamiento (González-Arratia, 2011).

Respecto a los niveles de resiliencia, una puntuación alta en los individuos refleja que, en caso de una situación de internamiento, esta podría favorecerlos en cuanto a presentar mayores características adaptativas a la institución y a su funcionamiento. Una puntuación baja sugiere que los individuos mostrarán indicios de mayor vulnerabilidad, puesto que cuentan con menos capacidades de adaptabilidad y son propensos a tener mayor conflicto en las relaciones interpersonales (González-Arratia, 2011).

Una puntuación moderada reflejará que existen habilidades individuales, pero que no se tiene clara necesidad de reforzarlas y que de ser así sí se beneficiarían mucho de esta condición. Por ello es importante enriquecer vínculos sociales y familiares, así como promover habilidades para la vida, sobre todo en ambientes penitenciarios (González-Arratia, 2011).

En cuanto a la variable sexo, en la muestra de adolescentes se encontraron diferencias estadísticamente significativas en el nivel de resiliencia; aquí las mujeres son quienes evidenciaron ser más resilientes, lo cual puede deberse a que el rol de la mujer en la sociedad es menos restrictivo en cuanto a mostrar sentimientos y tiende a buscar con mayor facilidad redes de apoyo ante situaciones adversas, ya que la percepción es más positiva que la que tienen los hombres favoreciendo la resiliencia.

Tener conocimiento de los factores de riesgo en individuos en internamiento propicia una intervención adecuada en ellos, puesto que a mayor conocimiento sobre eventos negativos, mayor probabilidad de actuar sobre ellos con anticipación para evitarlos y así cambiar las condiciones que lo pueden exponer a adquirir enfermedades o algún daño (Rutter, 1985 en González-Arratia, 2011). Al mismo tiempo, al reforzar y promover los factores de protección, se propiciará el desarrollo de la resiliencia.

Es importante ampliar las investigaciones referentes a aspectos psicológicos positivos de las personas privadas de la libertad (hombres y mujeres) más allá de la psicopatología, como puede ser desde la psicología positiva y resiliencia, y comenzar a romper estigmas respecto a este tipo de poblaciones.

Esto proporcionará un panorama más amplio en cuanto a la situación de vida en las instituciones penitenciarias favoreciendo diagnósticos más acertados y por ende manejar una intervención adecuada. De este modo se propiciaría que la reinserción sea un tema tangible y alcanzable para todas las personas privadas de la libertad fomentando la reintegración pertinente y pronta a la sociedad. 


\section{AnÁlisis PRospectivo}

En la actualidad el aumento en la incidencia delictiva ha provocado que los centros penitenciarios manifiesten diferentes problemáticas, como lo es la sobrepoblación, condiciones precarias de vida, circunstancias de salud desfavorables, abandono familiar, mayor demanda económica en el presupuesto de estos centros y la reincidencia delictiva (CNDH, 2013). Estas circunstancias se ven reflejadas como un problema de salud y seguridad pública en nuestro país.

Específicamente, en las instituciones penitenciarias la reincidencia delictiva se ha vuelto un foco de alerta, puesto que el eje primordial de intervención dentro de ellas se ha vuelto nulo y han quedado de lado sus objetivos primordiales: la reinserción y la readaptación a la sociedad retomando más bien una función meramente punitiva y de contención. Resulta importante considerar que esta disfuncionalidad institucional tiene que ver con la escasa información acerca de la verdadera dinámica de las personas que en ellas viven y con ello poder cambiar el paradigma respecto a sus características, así que un enfoque desde la psicología positiva dará pie a comenzar con una nueva alternativa, es decir, buscar más allá de la patología y dirigirse a las fortalezas humanas, entre ellas, una de suma importancia, la resiliencia (Seligman, 2003).

Esta investigación evidencia que los individuos en reclusión también presentan estas características permitiendo la creación de un antecedente teórico referente a estos contextos tan peculiares y así encentivar futuras investigaciones o propuestas, donde a partir de las fortalezas y características resilientes, así como por la confirmación de la importancia que representa en los individuos las redes de apoyo (González Arratia, 2011), se puedan proponer programas de intervención con un enfoque distinto que se adhieran a las reformas y leyes establecidas para los contextos penitenciarios.

\section{ReFerencias}

Becerra, S., Torres, G. y Ruiz, J. I. (2008). Estudio longitudinal y comparativo sobre la adaptación psicosocial a contextos carcelarios. Anuario de Psicología Jurídica Vol. 18. Colegio Oficial de Psicólogos de Madrid.

Bernard, B. (1999). Applications of resilience: Possibilities and promise. In Glantz \& J. Johnson (Ed.), Resilience and development: Positive life adaptation (pp. 269-277). New York: Plenum.

Bronfenbrenner, U. (1979). The ecology of human development (trad. cast. La ecología del desarrollo humano). Cambridge: Harvard University Press.

Bronfenbrenner, U. (2000). Ecological systems theory. In A. Kazdin (Ed.), Encyclopedia of Psychology Vol. 3 (pp. 129-133). Washington, D. C. American Psychological Association.

Córdova, A. (2006). Características de resiliencia en jóvenes usuariosyno usuarios de drogas (tesis doctoral). México: UNAM.

CNDH (Comisión Nacional de Derechos Humanos). (2013). Estadisticas del sistema penitenciario nacional. Disponible en http://www.cndh.org.mx/node/908131

González Arratia. L. F. N. I. (2007). Factores determinantes de la resiliencia en niños de la ciudad de Toluca (tesis no publicada de doctorado). Universidad Iberoamericana.

González Arratia, L. F. N. I. (2011). Resiliencia y personalidad en niños y adolescentes. Cómo desarrollarse en tiempos de crisis. México: Dirección de Difusión y Promoción de la Investigación y los Estudios Avanzados.

González Arratia, L. F. N. I. (2018). Autoestima, optimismo y resiliencia en niños en situación de pobreza. Revista Internacional de Psicología, 16(1), 1-119.

González Arratia, L. F. N. I., Valdez J. L. y Oudhof B. H. (2008). Resiliencia y optimismo como recurso psicológico para la salud en adolescentes. Presentado en el XXX Congreso CNEIP, Puerto Vallarta.

González Arratia, L. F. N. I, Valdez, J. L. y González E., S. (2011). Capítulo 5. Investigación en resiliencia: ¿Qué hemos aprendido?, en J. Moral, J. L. Valdez, L. F. N. I. González Arratia, Psicología y Salud (pp.157-168). México: Consorcio de Universidades Mexicanas. 
González Arratia, L. F. N. I. y Valdez, J. L. (2012). Optimismo-Pesimismo y Resiliencia en adolescentes de una universidad pública. CIENCIA ergo-sum, 19(3). Disponible en https://cienciaergosum.uaemex.mx/ article/view/7043

Grieco, D. (2012). Rasgos de personalidady caracteristicas resilientes en adultos en conflicto con la ley penal. Consultado el 23 de octubre de 2013. Disponible en http://imgbiblio.vaneduc.edu.ar/fulltext/files/TC106645. pdf [Accesado 23 octubre de 2013]

Grotberg, E. (2006). La resiliencia en el mundo de hoy. Cómo superar las adversidades. Barcelona: Gedisa.

Hernández, R., Fernández, C. y Baptista, P. (2003). Metodología de la investigación (seg. ed.). México: McGraw-Hill.

Hernández, M. G (2008). Resiliencia y recursos psicológicos (relaciones intrafamiliares y autoestima) en niños de la calle y en menores infractores en el Estado de México (tesis de licenciatura). Toluca: UAEM.

Hernández, R. (2009). Menores infractores en la frontera de México-Estados Unidos. Cultura e identidad frente al papel de las Instituciones. Estudios de casos 1996-2008. México: Porrúa

Herrera, M. C. y Expósito, F. (2010). Una vida entre rejas: aspectos psicosociales de la encarcelación y diferencias de género. Psychosocial Intervention, 19(3). Colegio Oficial de Psicólogos de Madrid.

INEGI (2013) Censo Nacional de Gobierno, Seguridad Pública y Sistema Penitenciario Estatales 2013. Disponible en https://www.inegi.org.mx/programas/cngspspe/2013/

Kaplan, H. B. (1999). Toward an understanding of resilience: A critical review of definitions and models. In M. Glantz \& J. Johnson (Ed.), Resilience and development: Positive life adaptation. New York: Plenum.

Lazarus, R. S. y Folkman, S. (1991). Estrés y procesos cognitivos. Barcelona: Martínez Roca.

Luthar, S.(1993). Annotation: methodological and conceptual issues in research on childhood resilience. Journal of Child Psychology and Psychiatry.

Masten, A. (1999). Resilience comes of age: Reflections of the past and outlooks for the next generation of researches. En M. Glantz y J. Johnson (Ed.). Resilience and Development: Positive Life Adaptation. New York. Plenum.

Masten, A., Best, K. y Garmezy, N. (1990). Resilience and development: Contributions from the study of children who overcome adversity. Development and Psychopathology, 2.

Mikulic, I. M. y Crespi, M. C. (2004). Contexto carcelario: un estudio de los estresores y las respuestas de afrontamiento en detenidos primaries y reincidentes. Facultad de Psicología-UBA Secretaría de Investigaciones. XII Anuario de Investigaciones.

Montelongo, D. (2013). Experiencia resiliente oncológica. Revista Psicología 2013. México: Producciones Gráficas.

Ramírez, C. (2008). Resiliencia y recursos psicológicos en hombres y mujeres que se encuentran en la escuela de reintegración social para adolescentes quinta del bosque (tesis de licenciatura). Toluca: UAEM.

Rubio, F. J. (2013). Aspectos psicosociológicos de las personas encarceladas y/o Excarceladas. Nómadas, 37, enero-junio. Madrid: Universidad Complutense de Madrid.

Saavedra, E. y Villalta, M. (2008). Medición de las características resilientes, un estudio comparativo en personas entre 15 y 45 años. Liberabit 14, 31-4’j0. Disponible en http://www.scielo.org.pe/pdf/liber/v14n14/ a05v14n 14.pdf

Saavedra, E. (2009). Jóvenes y resiliencia. Disponible en https://www.slideshare.net/RedUPRA/jvenes-y-resiliencia [Accesado 13 de octubre 2013]

Seligman, M. (2003). La auténtica felicidad. Byblos: España.

Vargas, K. (2010). Resiliencia y relaciones intrafamiliares en adultos sentenciados y procesados del C. P. R. S Santiaguito (tesis de licenciatura). Toluca: UAEM.

Vera, P. B.;, Carbelo B. B. y Vecina J. M. (2006). La experiencia traumática desde la psicología positiva: resiliencia y crecimiento postraumático, Papeles del psicólogo, 27(1).

\section{BY-NC-ND}


